\title{
Enumeration of Triangles in a Divisor Cayley Graph
}

Chalapathi, $\mathrm{T}^{1}$, Madhavi, $\mathrm{L}^{2 *}$ and Venkataramana, $\mathrm{S}^{3}$

${ }^{1}$ Department of Mathematics, J.B.Womens Engg. College, Tirupati, Andhra Pradesh, India

${ }^{2}$ Department of Applied Mathematics, Y.V.University, Kadapa, Andhra Pradesh, India

${ }^{3}$ Department of Mathematics, S.V.University, Tirupati, Andhra Pradesh, India

(*levakumadhavi@yahoo.in)

ABSTRACT

In this paper a new class of arithmetic Cayley graphs, namely, divisor Cayley graphs associated with the divisor function $d(n), n \geq 1$, an integer is introduced. It is shown that this graph is regular, hamiltonian, connected and not bipartite, and when $n$ is odd it is eulerian. The enumeration of triangles in this graph is also presented

Key words: Arithmetic graph, Cayley graph, Hamilton cycle, Fundamental triangle and Triangle.

[AMS (MOS) Subject Classification: 68R05]

\section{INTRODUCTION}

Nathanson (1980) was the pioneer in introducing the concepts of Number Theory, particularly the theory of congruences in Graph Theory, thus paving the way for the emergence of a new class of graphs, namely, arithmetic graphs. An arithmetic graph is the graph whose vertex set $V$ is the set of first $n$ positive integers $1,2,3, \ldots, n$ and two vertices $x$ and $y$ are adjacent if and only if $x+y \equiv z(\bmod n)$ where $z \in S$, a pre-assigned subset of $V$.

There is another class of graphs, called, Cayley graphs. A Cayley graphs is the graph whose vertex set $V$ is the set of elements of a finite $\operatorname{group}(X,$.$) and two vertices x$ and $y$ of $X$ are adjacent if and only if $x^{-1} y$ or $y^{-1} x$ is in some symmetric subset $S$ of $X$ ( a subset $S$ of a group $(X,$.$) is called symmetric subset of X$ if $s^{-1}$ is in $S$ for all $s$ in $S$ ). This Cayley graph is denoted by $G(X, S)$ and it is $|S|$-regular and contains $|X||S| / 2$ edges.(see pp 15,16,Madhavi, 2002). If the $\operatorname{group}(X,$.$) is the additive group \left(Z_{n}, \oplus\right)$ of integers $0,1,2, \ldots, n-1$ modulo $n$ and the symmetric set $S$ is associated with some arithmetic function, then the Cayley graph may be treated as an arithmetic graph and such graphs are called arithmetic Cayley graphs.

Dejter and Giudici (1995); Berrizabeitia and Giudici (1996) and others have studied the cycle structure of Cayley graphs associated with certain arithmetic functions. Madhavi and Maheswari $(2009,2010)$ studied the Hamilton cycles and triangles (the cycles of longest and 
shortest length) of the arithmetic Cayley graphs associated with Euler totient function $\varphi(n), n \geq 1$, an integer and quadratic residues modulo a prime $p$. The cycle structure of these graphs has many applications in Engineering and Communication Networks. Madhavi (2002) also studied the arithmetic Cayley graph associated with divisor function $d(n), n \geq 1$, an integer. This paper is devoted for the enumeration of triangles in these graphs. In this study we have followed Bondy and Murty (1979) for graph theory and Apostol (1989) for number theory terminology and notations not explained here.

\section{DIVISOR CAYLEY GRAPH AND ITS PROPERTIES}

In this section we introduce the notion of the divisor Cayley graph associated with the divisor function $d(n), n \geq 1$, an integer (Apostol, 1989) and briefly outline its basic properties that are given in Madhavi (2002).

\subsection{Definition}

Let $n \geq 1$ be an integer and let $S$ be the set of divisors of $n$. The set $S^{*}=\{s, n-s: s \in S\}$ is a symmetric subset of the additive abelian $\operatorname{group}\left(Z_{n}, \oplus\right)$ of integers modulo $n$. The Cayley graph of $\left(Z_{n}, \oplus\right)$ associated with the above symmetric subset $S^{*}$ is called the divisor Cayley graph and it is denoted by $G\left(Z_{n}, S^{*}\right)$. That is, the graph $G\left(Z_{n}, S^{*}\right)$ is the graph whose vertex set is $V=\{0,1,2, \ldots, n-1\}$ and the edge set $E$ is the set of all ordered pairs of vertices $a, b$ such that either $a-b \in S^{*}, o r, b-a \in S^{*}$.

\subsection{Example}

The divisor Cayley graphs $G\left(Z_{6}, S^{*}\right)$ and $G\left(Z_{9}, S^{*}\right)$ are given below.

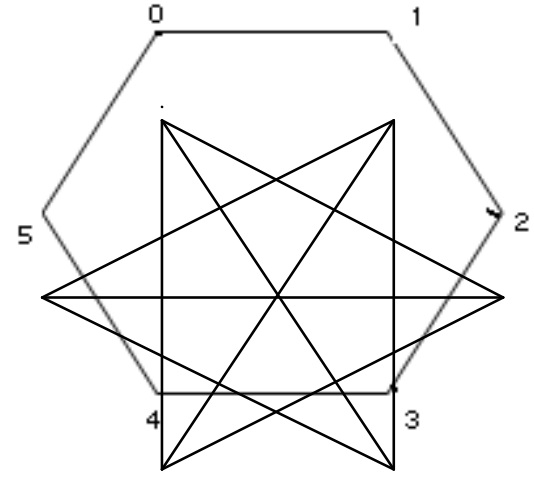

$G\left(Z_{6}, S^{*}\right) G\left(Z_{9}, S^{*}\right)$

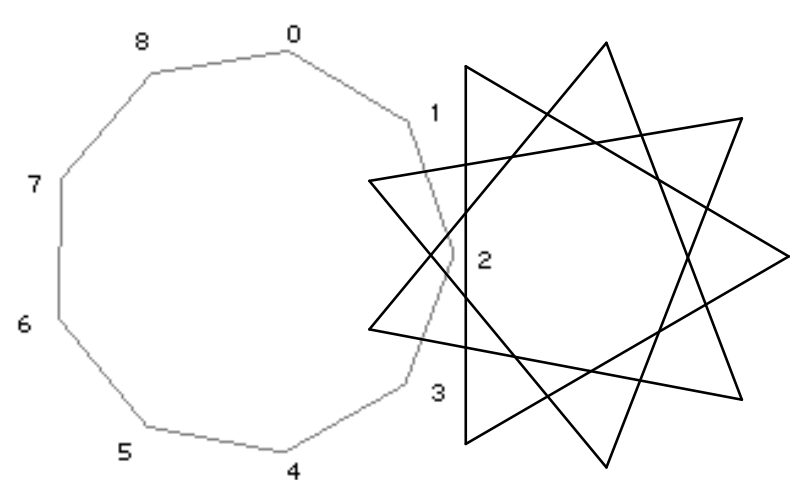


For $G\left(Z_{9}, S^{*}\right), S=\{1,3\}, S^{*}=\{1,3,8,6\}$. There is an edge between 0 and $1 ; 0$ and $3 ; 0$ and 6 ; 0 and 8 since $1-0=1,3-0=3,6-0=6,8-0=8$ are elements in $S^{*}$. Similarly there is an edge between 1 and $2 ; 1$ and $4 ; 1$ and $7 ; 2$ and $3 ; 2$ and $5 ; 2$ and $8 ; 3$ and $4 ; 3$ and $6 ; 4$ and $5 ; 4$ and $7 ; 5$ and $6 ; 5$ and $8 ; 6$ and $7 ; 7$ and 8 . But there is no edge between 0 and 4; 0 and 5;0 and 7 since $4-0=4,5-0=5$ and $7-0=7$ are not in $S^{*}$. Similarly there is no edge between 1 and $5 ; 1$ and $6 ; 1$ and $8 ; 2$ and $4 ; 2$ and $6 ; 2$ and $7 ; 3$ and $5 ; 3$ and $7 ; 3$ and $8 ; 4$ and $6 ; 4$ and $8 ; 5$ and $7 ; 6$ and 8 .

In a similar way the graph $G\left(Z_{6}, S^{*}\right)$ can be drawn.

The following lemma is immediate since the graph $G\left(Z_{n}, S^{*}\right)$ is the Cayley graphofthe group $\left(Z_{n}, \oplus\right)$ with respect to the symmetric set $S^{*}$. This is useful in finding the basic properties of the divisor Cayley graph $G\left(Z_{n}, S^{*}\right)$.

\subsection{Lemma}

The graph $G\left(Z_{n}, S^{*}\right)$ is $\left|S^{*}\right|$ - regular and the number of edges in it is $n|S| / 2$.

\subsection{Lemma}

(a) Degree of each vertex in $G\left(Z_{n}, S^{*}\right)$ is odd if and only if $n$ is even.

(b) Degree of each vertex in $G\left(Z_{n}, S^{*}\right)$ is even if and only if $n$ is odd.

(c) $G\left(Z_{n}, S^{*}\right)$ is Eulearian if and only if $\mathrm{n}$ is odd.

Proof: Here $S^{*}=\{d, n-d: d$ is a divisor of $n\}$. Let us pair the elements of $S^{*}$ as $(d, n-d)$, $d \mid n$.

For some $d \mid n, d=n-d$ if and only if $n=2 d$, or , $n$ is even. In this case $S^{*}$ contains the distinct ordered pairs $(d, n-d), d \neq n-d$ and $\frac{n}{2}$ so that $\left|S^{*}\right|$ is odd.

On the other hand $d \neq n-d$ for all $d \mid n$ if and only $n \neq 2 d$ for all $d \mid n$, or , $n$ is odd. In this case $\left|S^{*}\right|$ is even since the elements of each pair $(d, n-d), d \mid n$ in $S^{*}$ are distinct

By the Lemma 2.3 the degree of each vertex of $G\left(Z_{n}, S^{*}\right)$ is $\left|S^{*}\right|$. From these (a) and (b) follow. Part (c) follows from the fact that a connected graph is eulerian if and only if the degree of each of its vertex is even (see pp 51,52 of Bondy and Murty,1976). 


\subsection{Lemma}

The Graph $G\left(Z_{n}, S^{*}\right)$ is hamiltonian and connected but not bipartite.

Proof: For $i \in Z_{n},(i+1)-i=1 \in S^{*}$. So $C=(1,2,3, \ldots, n, 1)$ is a Hamilton cycle of length $n$. So $G\left(Z_{n}, S^{*}\right)$ is Hamiltonian and hence connected.

Suppose $n$ is odd. Then the cycle $C$ is an odd cycle.

On the other hand if $n$ is even, then $1,2 \in S^{*}$. So $n-1, n-2 \in S^{*}$. These show that $(0,1, n-1,0)$ is a 3 -cycle, which is an odd cycle in $G\left(Z_{n}, S^{*}\right)$. In either case $G\left(Z_{n}, S^{*}\right)$ contain an odd cycle and thus it is not bipartite, since a graph is bipartite if and only if it contains no odd cycles (see pp 14, 15, Bondy and Murty (1976)).

\subsection{Definition}

The Cycle $(0,1,2, \ldots, n-1,0)$ is called the outer Hamilton Cycle.

\subsection{Lemma}

If $\mathrm{n}$ is a prime then the graph $G\left(Z_{n}, S^{*}\right)$ is the outer Hamilton Cycle.

Proof: Suppose that $n$ is a prime. Then 1 is the only divisor of $n$ other than $n$, so that $S^{*}=\{1, n-1\}$. Hence by the Lemma 2.3 , the graph $G\left(Z_{n}, S^{*}\right)$ is 2 -regular and each vertex is of degree 2 . So the only edges in $G\left(Z_{n}, S^{*}\right)$ are $(i, i+1)$ for $0 \leq i \leq n-1$ and $G\left(Z_{n}, S^{*}\right)$ is the outer Hamilton cycle.

\subsection{Example}

For the prime 11 the divisor Cayley graph $G\left(Z_{11}, S^{*}\right)$ is given below.

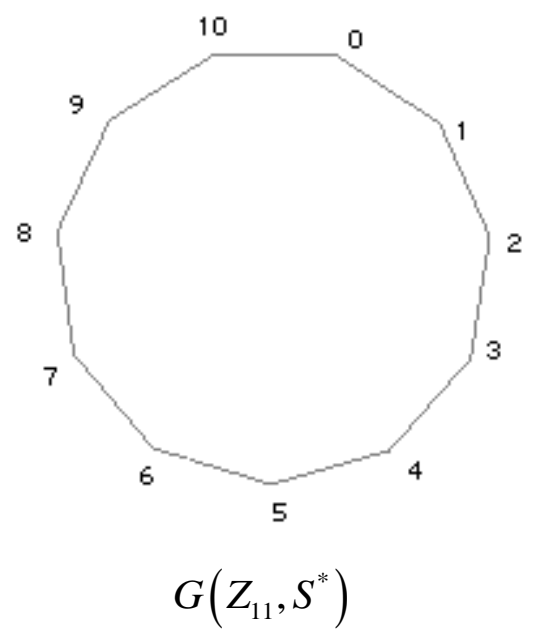




\section{ENUMERATION OF TRIANGLES IN A DIVISOR CAYLEY GRAPH}

In this section we present a criterian for the enumeration of triangles in the divisor Cayley graph $G\left(Z_{n}, S^{*}\right)$.

\subsection{Definition}

i. In a graph a 3-cycle is called a triangle.

ii. For the vertices $a, b, c$ in a graph $G$ the triangle formed by the distinct vertices $a, b, c$ is denoted by the triad $(a, b, c)$.

iii. In $G\left(Z_{n}, S^{*}\right)$ the triangle $(0, a, b)$ iscalled a fundamental triangle and it is denoted by $\Delta_{a b}$.

\subsection{Remark}

The triangle $\Delta_{a b}$ is a fundamental triangle in $G\left(Z_{n}, S^{*}\right)$ if and only if $a-0 \in S^{*}, b-0 \in S^{*}$ and $b-a \in S^{*}, o r, a, b, b-a \in S^{*}$. In the same way $(0, b, a)$ is a triangle in $G\left(Z_{n}, S^{*}\right)$ if and only if $a, b, b-a \in S^{*}$. By the definition of adjacency in $G\left(Z_{n}, S^{*}\right)$ the triangle $\Delta_{a b}$ is same as $\Delta_{b a}$.

The following lemma gives a formula for the number of fundamental triangles in $G\left(Z_{n}, S^{*}\right)$ corresponding to a fixed element in $S^{*}$. This is useful in finding the total number of fundamental triangles in $G\left(Z_{n}, S^{*}\right)$.

\subsection{Lemma}

For a given $a \in S^{*}$ the number of fundamental triangles $\Delta_{a b}$ in $G\left(Z_{n}, S^{*}\right)$ is $\left|S^{*} \cap\left(a+S^{*}\right)\right|$ where $a+S^{*}=\left\{a+s: s \in S^{*}\right\}$.

Proof: Let $a \in S^{*}$.For any $b \in Z_{n}$ suppose that $(0, a, b)$ is a fundamental triangle. Then by the Remark 3.2, $a, b, b-a \in S^{*}$,or, $a, b \in S^{*}$ and $b \in a+S^{*}$, or , $a \in S^{*}$ and $b \in S^{*} \cap\left(a+S^{*}\right)$.

On the other hand let $b \in S^{*} \cap\left(a+S^{*}\right)$. Then $b \in S^{*}$ and $b \in a+S^{*}$,or, $b \in S^{*}$ and $b-a \in S^{*}$ That is, $a \in S^{*}, b \in S^{*}$ and $b-a \in S^{*}$. Again by the Remark 3.2, $(0, a, b)$ is a fundamental triangle in $G\left(Z_{n}, S^{*}\right)$. 
That is, for a given $a \in S^{*}$ and for each $b \in S^{*} \cap\left(a+S^{*}\right)$ the triad $(0, a, b)$ is a fundamental triangle in $G\left(Z_{n}, S^{*}\right)$ so that the number of fundamental triangles $\Delta_{a b}$ in $G\left(Z_{n}, S^{*}\right)$ is $\left|S^{*} \cap\left(a+S^{*}\right)\right|$.

\subsection{Example}

For the Cayley Divisor Graph $G\left(Z_{10}, S^{*}\right)$ the fundamental triangles are determined below.

Here $S=\{1,2,5\}$ and $S^{*}=\{1,2,5,8,9\}$.

i. For $a=1,1+S^{*}=\{2,3,6,9,0\}$, and $S^{*} \cap\left(1+S^{*}\right)=\{2,9\}$ so that $b=2$,or, 9 and $\left|S^{*} \cap\left(1+S^{*}\right)\right|=2$. So there are two fundamental triangles corresponding to $1 \in S^{*}$, namely $\Delta_{12}=(0,1,2)$ and $\Delta_{19}=(0,1,9)$.

ii. For $a=2,2+S^{*}=\{3,4,7,0,1\}$ and $S^{*} \cap\left(2+S^{*}\right)=\{1\}$ so that $b=1$ and $\left|S^{*} \cap\left(2+S^{*}\right)\right|=1$. This shows that there is only one fundamental triangle $\Delta_{21}=(0,2,1)$.

iii. For $a=5,5+S^{*}=\{6,7,0,3,4\}$ and $S^{*} \cap\left(5+S^{*}\right)=\phi$.This shows that there is no fundamental triangle corresponding to $5 \in S^{*}$.

iv. For $a=8,8+S^{*}=\{9,0,3,6,7\}$ and $S^{*} \cap\left(8+S^{*}\right)=\{9\}$ so that there is only one fundamental triangle $\Delta_{89}=(0,8,9)$ corresponding to $8 \in S^{*}$.

v. For $a=9,9+S^{*}=\{0,1,4,7,8\}$ and $S^{*} \cap\left(9+S^{*}\right)=\{1,8\}$. This shows that there are two fundamental triangles corresponding to $9 \in S^{*}$ and they are $\Delta_{91}=(0,9,1)$ and $\Delta_{98}=(0,9,8)$.

\subsection{Remark}

Since $\Delta_{a b}=\Delta_{b a}$ for $a, b \in S^{*}$, the distinct fundamental triangles of the graph $G\left(Z_{10}, S^{*}\right)$ are $\Delta_{12}, \Delta_{19}$ and $\Delta_{89}$ which are represented by thick lines in the figure given below. 


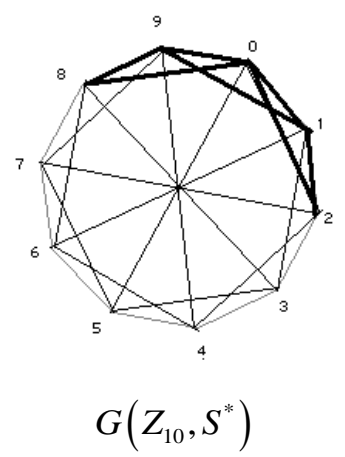

The following lemma gives a formula for the total number of fundamental triangles in $G\left(Z_{n}, S^{*}\right)$.

\subsection{Lemma}

The number of fundamental triangles in $G\left(Z_{n}, S^{*}\right)$ is $\frac{1}{2} \sum_{a \in S^{*}}\left|S^{*} \cap\left(a+S^{*}\right)\right|$.

Proof: By the Lemma 3.3, for a given $a \in S^{*}$ the number of fundamental triangles in $G\left(Z_{n}, S^{*}\right)$ is $\left|S^{*} \cap\left(a+S^{*}\right)\right|$. So the total number of fundamental triangles in $G\left(Z_{n}, S^{*}\right)$ is $\sum_{a \in S^{*}}\left|S^{*} \cap\left(a+S^{*}\right)\right| \cdot$

However for $a, b \in S^{*}$ we have seen that $\Delta_{a b}$ and $\Delta_{b a}$ represent the same fundamental triangle. So the number of fundamental triangles in $G\left(Z_{n}, S^{*}\right)$ is $\frac{1}{2} \sum_{a \in S^{*}}\left|S^{*} \cap\left(a+S^{*}\right)\right|$.

We now prove the main theorem which gives the total number of trianglesin $G\left(Z_{n}, S^{*}\right)$. Using this formula one can find the number of triangles in $G\left(Z_{n}, S^{*}\right)$ for any positive integer $n$.

\subsection{Theorem}

The number of triangles in the Cayley Divisor Graph $G\left(Z_{n}, S^{*}\right)$ is $\frac{1}{6}\left|Z_{n}\right| \sum_{a \in S^{*}}\left|S^{*} \cap a+S^{*}\right|$.

Proof: The graph $G\left(Z_{n}, S^{*}\right)$ is vertex symmetric, that is, for any $g \in G$ there is an automorphism $\theta$ of $G\left(Z_{n}, S^{*}\right)$ given by

$\theta(x)=g+x$ for all $x \in G$.

Clearly, $\theta(0)=0+g=g$. 
Since an automorphism preserves incidence, that is, it maps adjacent vertices into adjacent vertices and non -adjacent vertices into non -adjacent vertices, the fundamental triangle $(0, a, b)$ is taken to the triangle $(g, g+a, g+b)$.

So for each $g \in G$ the number of triangles of the form $(g, x, y)$ is also $\frac{1}{2} \sum_{a \in S^{*}}\left|S^{*} \cap\left(a+S^{*}\right)\right|$ and the total number of triangles in $G\left(Z_{n}, S^{*}\right)$ is given by $\frac{1}{2}\left|Z_{n}\right| \sum_{a \in S^{*}}\left|S^{*} \cap\left(a+S^{*}\right)\right|$.

However, in the above enumeration each triangle in $G\left(Z_{n}, S^{*}\right)$ is counted thrice, namely, once, by each of its three vertices. So the total number of distinct triangles in $G\left(Z_{n}, S^{*}\right)$ is is $\frac{1}{6}\left|Z_{n}\right| \sum_{a \in S^{*}}\left|S^{*} \cap\left(a+S^{*}\right)\right|$.

\subsection{Example}

Consider the Cayley Divisor Graph $G\left(Z_{15}, S^{*}\right)$.

Here $Z_{15}=\{0,1,2,3,4,5,6,7,8,9,10,11,12,13,14\}, S=\{1,3,5\}$ and $S^{*}=\{1,3,5,14,12,10\}$.

For $a \in S^{*}$, the sets $a+S^{*}, S^{*} \cap\left(a+S^{*}\right)$ and $\left|S^{*} \cap\left(a+S^{*}\right)\right|$ are listed below:

\begin{tabular}{|c|c|c|c|}
\hline$a$ & $a+S^{*}$ & $S^{*} \cap\left(a+S^{*}\right)$ & $\left|S^{*} \cap\left(a+S^{*}\right)\right|$ \\
\hline 1 & $\{2,4,6,0,13,11\}$ & $\phi$ & 0 \\
\hline 3 & $\{4,7,8,2,0,13\}$ & $\phi$ & 0 \\
\hline 5 & $\{6,8,10,4,2,0\}$ & $\{10\}$ & 1 \\
\hline 14 & $\{0,2,4,13,11,9\}$ & $\phi$ & 0 \\
\hline 12 & $\{13,0,2,11,9,7\}$ & $\phi$ & 1 \\
\hline 10 & $\{11,13,0,9,7,5\}$ & $\{5\}$ & \\
\hline
\end{tabular}

Hence the number of triangles in $G\left(Z_{15}, S^{*}\right)$ $=\frac{1}{6}\left|Z_{15}\right| \sum_{a \in S^{*}}\left|S^{*} \cap\left(a+S^{*}\right)\right|=\frac{15}{6}[0+0+1+0+0+1]=5$.

The five triangles $(0,5,10),(1,6,11),(2,7,12),(3,8,13),(4,9,14)$ in $\quad G\left(Z_{15}, S^{*}\right)$ are represented by thick lines in its graph below. 




\subsection{Example}

Consider the Cayley divisor graph $G\left(Z_{12}, S^{*}\right)$. Here $S^{*}=\{1,2,3,4,6,11,10,9,8\}$. For $a \in S^{*}$ the sets $a+S^{*}, S^{*} \cap\left(a+S^{*}\right)$ and $\left|S^{*} \cap\left(a+S^{*}\right)\right|$ are given by the following table :

\begin{tabular}{|l|l|l|l|}
\hline$a$ & $a+S^{*}$ & $S^{*} \cap\left(a+S^{*}\right)$ & $\left|S^{*} \cap\left(a+S^{*}\right)\right|$ \\
\hline 1 & $\{2,3,4,5,7,12,11,10,9\}$ & $\{2,3,4,11,10,9\}$ & 6 \\
\hline 2 & $\{3,4,5,6,8,1,0,11,10\}$ & $\{3,4,6,8,1,11,10\}$ & 7 \\
\hline 3 & $\{4,5,6,7,9,2,1,0,11\}$ & $\{4,6,9,2,1,11\}$ & 6 \\
\hline 4 & $\{5,6,7,8,10,3,2,1,0\}$ & $\{6,8,10,3,2,1\}$ & 6 \\
\hline 6 & $\{7,8,9,10,0,5,4,3,2\}$ & $\{8,9,10,4,3,2\}$ & 6 \\
\hline 11 & $\{0,1,2,3,5,10,9,8,7\}$ & $\{1,2,3,10,9,8\}$ & 6 \\
\hline 10 & $\{11,0,1,2,4,9,8,7,6\}$ & $\{11,1,2,4,9,8,6\}$ & 7 \\
\hline 9 & $\{10,11,0,1,3,8,7,6,5\}$ & $\{10,11,1,3,8,6\}$ & 6 \\
\hline 8 & $\{9,10,11,0,2,7,6,5,4\}$ & $\{9,10,11,2,6,4\}$ & 6 \\
\hline
\end{tabular}

So the number of fundamental triangles in $G\left(Z_{12}, S^{*}\right)$

$=\frac{1}{2} \sum_{a \in S^{*}}\left|S^{*} \cap\left(a+S^{*}\right)\right|=\frac{1}{2}[6+7+6+6+6+6+7+6+6]=\frac{56}{2}=28$. 




From the graph $G\left(Z_{12}, S^{*}\right)$ one can observe that the 28 distinct fundamental triangles with 0 as one vertex are $(0,1,3),(0,2,4),(0,3,6),(0,4,8),(0,6,9),(0,8,10),(0,9,11)$, $(0,1,4),(0,2,6),(0,3,9),(0,4,10),(0,6,10),(0,8,11)$, $(0,1,9),(0,2,8),(0,3,11)$, $(0,1,10),(0,2,10)$, $(0,1,11),(0,2,11)$.

These are represented by thick lines in above figure.

By the Theorem 3.7 the number of triangles in $G\left(Z_{12}, S^{*}\right)$ $=\frac{1}{6}\left|Z_{12}\right| \sum_{a \in S^{*}}\left|S^{*} \cap\left(a+S^{*}\right)\right|=\frac{12}{6}[6+7+6+6+6+6+7+6+6]=2 \times 56=112$.

\subsection{Remark}

Observe that $G\left(Z_{15}, S^{*}\right)$ has only 5 triangles where as $G\left(Z_{12}, S^{*}\right)$ has 112 triangles. Since $15=3 \times 5$ and $12=2^{2} \times 3,12$ has more number of divisors than 15 . It will be interesting to investigate the relationship between the number of divisors of $n$ and the number of triangles in $G\left(Z_{n}, S^{*}\right)$.

\section{CONCLUSION}

In Madhavi and Maheswari $(2009,2010)$ the enumeration of triangles in Cayley graphs associated with the set of quadratic residues modulo $p$ and the Euler function $\varphi(n), n \geq 1$, a 
positive integer are obtained. In determining these triangles the properties of the multiplicative structure of the $\operatorname{ring}\left(Z_{n}, \oplus, \otimes\right)$ is also used. In this paper this aspect is not used in determining the number of triangles in divisor Cayley graphs. Only the fact that $\left(Z_{n}, \oplus\right)$ is a group is used. Since a Cayley graph is associated with a group $(X, \bullet)$ and its symmetric $S$, the authors have investigated whether the techniques used in this paper may be applied to general Cayley graphs in obtaining the number of triangles. These aspects will be dealt with separately.

\section{ACKNOWLEDGEMENT}

The authors express their thanks to Prof. L. Nagamuni Reddy for his suggestions during the preparation of this paper.

\section{REFERENCES}

Apostol, T. M. 1989. Introduction to Analytical Number Theory. Springer International Student First Edition.

Brrizbeitia, P \& Gaudici, R.E.1996. Counting pure k-cycles in sequences of Cayley graphs, Discrete math., 149:11-18.

Bondy, J.A \& Murty, U.S.R. 1976. Graph Theory with Application, Macmillan, London. 5152.

Dejter. I \& Giudici. R. E. 1995. On unitary Cayley graphs. JCMCC, 18: 121-124.

Madhavi, L. 2002. Studies on Domination Parameters and Enumeration of cycles in some arithmetic grpahs,Ph.D Thesis, Sri Venkateswara University, Tirupati, India, 50-83.

Madhavi, L \& Maheswari, B. 2009. Enumeration of Traingles and Hamilton cycles in Quadratic Residue Cayley graphs. Chamchuri Journal of Mathematics, 1: 95-103.

Madhavi, L \& Maheswari,B. 2010. Enumeration of Hamilton cycles and Triangles in Euler Totient Cayley graphs. Graph Theory Notes of NewYork,LIX:28-31.

Nathanson \& Melvyn, B. 1980. Connected components of arithmetic graphs, Monat. fur. Math, 29: $219-220$. 